\title{
Comparative Analysis of Probability of Failure Determination Using Weibull Distribution and Generic Failure Frequencies on Heat Exchanger Tube Bundles Based on API 581
}

\author{
D.G.S. Prayoga ${ }^{1}$, Dwi Priyanta ${ }^{2}$, Nurhadi Siswantoro ${ }^{3}$
}

\begin{abstract}
RBI analysis for heat exchanger tube has been explained by API 581. Two types of PoF determination using statistical data is provided to determine the PoF category. Both Weibull parameter and generic failure frequencies are the methods that will be analysis in this present study. PoF determination using Weibull distribution gives the exponential value of PoF in a certain of time. This method only consider failure modes and the failure rate of heat exchanger tube without considering possible active damage mechanisms in the tube. However PoF determination using generic failure frequencies gives more complex consideration. It considers not only failure and possible active damage mechanism, but also the effect of management system facilities of the heat exchanger on the integrity of the plant.
\end{abstract}

Keywords: RBI analysis, heat exchanger, Weibull distribution, generic failure frequencies

\section{INTRODUCTION}

$\mathrm{H}_{\mathrm{e}}$ eat exchanger is an equipment which usually used to transfer heat from one medium to another. It is also the one of critical equipment in the oil and gas processing facility. Commonly, heat exchangers are categorized in three main types, i.e. "shell and tube", "plat type", and "air cooled".

Failure of tubes from a heat exchanger in offshore platform had been studied by many researchers. Corte et al [1] explained that the AISI 321 tubes of the heat exchanger failed due to high chloride content of the cooling water which cause stress corrosion cracking (SCC). Peltola and Lindgren [2] also explained that the leakage of the heat exchanger was caused by ant-nest corrosion in the copper tubes. Therefore, there are many types of prevention action to minimize the failure on heat exchanger. One of them is conducting risk assessment.

Risk based inspection (RBI) is the one of risk assessment methodology which optimized inspection plan on pressurized equipment based on their risk category. There are many types of equipment that was covered by RBI based on API 580, i.e. pressure vessels, process piping, storage tanks, rotating equipment, boiler and heaters, heat exchangers, and pressure relief devices [3].

Risk based approach basically explained by using risk category. Risk is determined by combination of the incident probability and its consequences. Thus, to carry

${ }^{1}$ D.G.S. Prayoga, Department of Corrosion Engineering, Universitas Indonesia, Jakarta, Indonesia,Email : dharmagita@gmail.com

${ }^{2}$ Dwi Priyanta, Department of Marine Engineering, Institut Teknologi Sepuluh Nopember, Surabaya 60111, Indonesia,Email : priyanta@its.ac.id

${ }^{3}$ Nurhadi Siswantoro, Department of Marine Engineering, Institut Teknologi Sepuluh Nopember, Surabaya 60111, Indonesia,Email : nurhadi@ne.its.ac.id out RBI analysis for each equipment, the probability of failure $(\mathrm{PoF})$ and consequences of failure $(\mathrm{CoF})$ are assessed separately [4]. API 581 has developed the determination of RBI analysis on heat exchanger. The purpose of this analysis is to optimize heat exchanger bundle inspection and replacement cycles and also to minimize the annual operating and maintenance costs of heat exchanger bundles [5]. However, to conduct RBI assessment on heat exchanger, PoF and $\mathrm{CoF}$ determination should consider any input parameters and assumptions, including all the fluid properties and conditions under which the exchanger is expected to operate [6]. The present work is determining comparison between PoF determination using Weibull distribution and using generic failure frequencies based on API 581.

\section{PROBABILITY OF FAILURE DETERMINATION}

Based on RBI definition, failure that was occurred on heat exchanger may be defined as a tube leak due to some damage mechanisms, such as general corrosion, erosion, vibration, etc. API 581 explained that bundle failure is able to be estimated by assuming the bundle will be replaced at $75 \%$ of the bundle life. The degraded condition assumption allows consideration for remaining life of the bundle when replaced prior to actual tube failure. Therefore, in order to determine probability of tube failure, there were two type of PoF determination, i.e. using Weibull distribution and generic failure frequencies (gff).

\section{II.1 Weibull Distribution}

The API 581 methodology for heat exchangers RBI analysis, there are two parameters that must firstly be obtained for Weibull distribution, i.e. scale parameter $(\eta)$ and shape parameter $(\beta)$. The PoF for a heat exchanger bundle at a certain time may be expressed:

$$
P o F_{\text {tube }}^{w b l}=1-\exp \left[-\left(\frac{t}{\eta}\right)^{\beta}\right]
$$


In order to determine the value of $\eta$, Offshore and Onshore Reliability Data (OREDA) will be used in this work. OREDA contains the failure frequency data for different process equipment that usually used in onshore and offshore oil and gas industry. Table 1 describe the selected failure modes and failure rates for shell and tube heat exchanger for this current work that was taken from OREDA.

TABLE 1SELECTED FAILURE MODES FOR HEAT EXCHANGER FAILURE RATE FROM OREDA

\begin{tabular}{|c|c|}
\hline Failure modes & $\begin{array}{c}\text { Mean Failure Rate } \\
\left(10^{6} \text { hours }\right)\end{array}$ \\
\hline External leakage-Process medium & 1.72 \\
\hline External leakage-Utility medium & 1.72 \\
\hline Insufficient heat transfer & 0.85 \\
\hline Internal leakage & 0.85 \\
\hline Plugged/Choked & 1.72 \\
\hline Total & 6.86 \\
\hline
\end{tabular}

Therefore, failure rate per year (FRtube) may become:

$$
\begin{aligned}
& F R_{\text {tube }}=\frac{\text { meanfailurerate }}{10^{6}} \times 8760 \text { hours } \\
& F R_{\text {tube }}=0.06 \text { peryear }
\end{aligned}
$$

The mean time to failure (MTTF) may be calculated:

$$
\begin{aligned}
M T T F & =\frac{1}{F R_{\text {tube }}} \\
M T T F & =16.64 \text { years }
\end{aligned}
$$

The value of MTTF may be used to determine the value of $\eta$. The MTTF value will be converted into a Weibull curve using a $\beta$ value of 3.0 [5]. Therefore, the $\eta$ parameter can be calculated using the gamma function (Г):

$$
\begin{aligned}
& \eta=\frac{M T T F}{\Gamma\left[1+\frac{1}{\beta}\right]} \\
& \eta=18.63
\end{aligned}
$$

Therefore, the PoF for heat exchanger bundle which is assumed already in service for 20 years,

$$
\begin{aligned}
& P o F_{\text {tube }}^{w b l}=1-\exp \left[-\left(\frac{20}{18.63}\right)^{3}\right] \\
P o F_{\text {tube }}^{w b l} & =0.71
\end{aligned}
$$

Figure 1 shows the exponential value of PoF from the selected failure mode and failure rate using Weibull distribution. From the graph, it is shown that the PoF will increase every year exponentially. It means that the failure of tube will be grow faster year by year. Based on that statement, the user should determine the threshold value of PoF that will be reference inspected time on RBI analysis. API 581 has categorized numerical values associated with PoF for heat exchanger bundles that showed in the Table 2. The $P o F_{t u b e}^{w b l}$ is categorized as category " 5 ".

\section{II.2 Generic Failure Frequencies (gff)}

API 581 also determined the PoF based on one or a combination of the following methods, i.e. structural reliability models, statistical models based on generic data, and expert judgment. Combination of those methods is used to evaluate the PoF in terms of a generic failure frequency (gff) and damage factor (Df).

The gff is estimated using any records from all plants within a company, literature, and from commercially reliability data bases [5]. The PoF for a heat exchanger bundles is computed using:

$$
P o F_{\text {tube }}^{g f f}=g f f . D_{f}(t) \cdot F_{M S}
$$

API 581 explained that the adjustment factors on the gff reflect differences between damage mechanisms and the reliability management processes within a plant. Equation (5) shows that the damage factor (Df) and management system factor (FMS) are adjusted factor for gff. The Df adjusts gff based on the active damage mechanism, inspection data and the effectiveness of past and future inspections. The FMS adjusts gff based on the effect of management system facilities on the integrity of the plant [5].

The gff determination using recommended list of gff which is provided by API 581. Table 3 shows the suggested gff for tube side of heat exchanger. Based on Table 3, the gff total that was used in this present works:

$$
g f f_{\text {total }}=3.06 E-05
$$

As mention above, The Df adjusts the gff value based on the active damage mechanism, inspection data and the effectiveness of past and future inspections. Schwartz explained that there are four types of heat exchanger failures that can occur: mechanical, chemically induced corrosion, combination of mechanical and chemically induced corrosion, and scale, mud and algae fouling [7]. Al-Baldawi and Abdullah explained the tube damage mechanisms in feed water heater are uniform or general corrosion, crevice and pitting corrosion, galvanic corrosion, erosion corrosion, de-alloying corrosion, impingement attack and stress corrosion cracking [8]. 
The possible damage mechanisms for tube stainless steel based on API 571 was stress corrosion cracking (SCC), erosion-corrosion, vibration-induced fatigue and general corrosion [9].

Therefore, the suggested active damage mechanism for tube heat exchanger that will be used in this works are general corrosion that will be categorized as thinning mode $\left(D_{f}^{\text {thin }}\right)$, and stress corrosion cracking that will be categorized as SCC mode $\left(D_{f}^{S C C}\right)$. The Df for thinning may be determined by [5]:
For this present work, $D_{f}^{\text {thin }}$ value is 0.1 because we assume there is no basic component data required for analysis based on API 581-Table 4.1 such as measured thickness, corrosion allowance, pressure, temperature, material specification, etc.

The Df for stress corrosion cracking $\left(D_{f}^{C l-S C C}\right)$ may be determined by:

$$
D_{f}^{C l-S C C}=D_{f B}^{C l S C C} \cdot(\max [a g e, 1.0])^{1.1}
$$

$D_{f}^{\text {thin }}=\max \left[\left(\frac{D_{f B}^{T h i n} \cdot F_{I P} \cdot F_{D L} \cdot F_{W D} \cdot F_{A M} \cdot F_{S M}}{F_{O M}}\right), 0.1\right]$

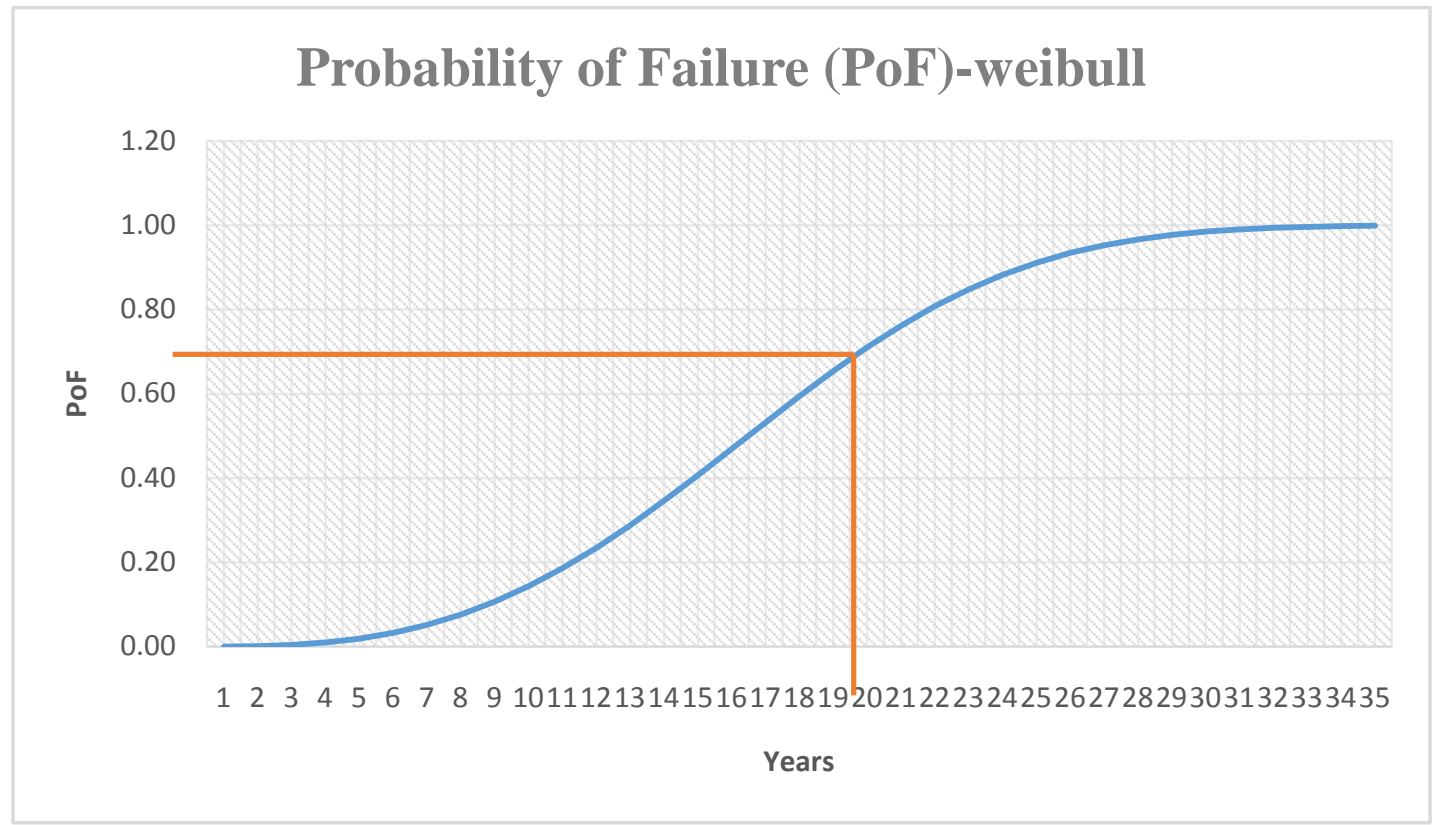

Figure 1. Probability of Failure (PoF) graph based on selected failure mode and failure rate from OREDA

TABLE 2POF CATEGORY FOR HEAT EXCHANGER BUNDLES BASED ON API 581

\begin{tabular}{c|c}
\hline & Probability Category \\
\hline Category & Range \\
\hline 1 & PoF $\leq 0.1$ \\
\hline 2 & $0.1 \leq$ PoF $\leq 0.2$ \\
\hline 3 & $0.2 \leq$ PoF $\leq 0.3$ \\
\hline 4 & $0.3 \leq$ PoF $\leq 0.5$ \\
\hline 5 & $0.5 \leq$ PoF $\leq 1.0$ \\
\hline
\end{tabular}

TABLE 3SUGGESTED GFF FOR TUBE SIDE OF HEAT EXCHANGER

\begin{tabular}{|c|c|c|c|c|c|c|c|}
\hline \multirow{2}{*}{$\begin{array}{l}\text { Equipment } \\
\text { Type }\end{array}$} & \multirow{2}{*}{$\begin{array}{c}\text { Component } \\
\text { Type }\end{array}$} & \multicolumn{4}{|c|}{ gff as a Function of Hole Size (failures/year) } & \multirow{2}{*}{$\begin{array}{c}\text { gff total } \\
\text { (failures/year) }\end{array}$} & \multirow{2}{*}{ Remarks } \\
\hline & & Small & Medium & Large & Rupture & & \\
\hline Heat Exchanger & HEXTS & 8.00E-06 & $2.00 \mathrm{E}-05$ & $2.00 \mathrm{E}-06$ & $6.00 \mathrm{E}-07$ & 3.06E-05 & Tube \\
\hline
\end{tabular}


TABLE 4DETERMINATION OF SEVERITY INDEX-CLSCC

\begin{tabular}{cc}
\hline Susceptibility & Severity Index-S \\
\hline High & 5000 \\
\hline Medium & 500 \\
\hline Low & 50 \\
\hline None & 1 \\
\hline
\end{tabular}

For this present work, we used the heat exchanger data:

a. The age of tube is 20 years

b. There was no inspection for 20 years

c. The operating temperatures is $150{ }^{\circ} \mathrm{C}$

d. Concentration of the chloride ion is more than 100 ppm with $\mathrm{pH} 3$

Therefore, we able to determine the base DF for ClSCC $\left(D_{f B}^{C l-S C C}\right)$ using those assumptions. The susceptibility to cracking as a function of chloride ion based on API 581-Table 12-2, may be determined as "High".

Based on susceptibility to cracking category, the severity index (SVI) may have value of 5000. Therefore, the $D_{f B}^{C l-S C C}$ factor with no inspection data and SVI value of 5000, may have a value of 5000 when it is plotted on Table 5 below.

The escalation in the Df based on the equation (7) may have value:

$$
\begin{array}{r}
D_{f}^{C l-S C C}=5000 \cdot(\max [20,1.0])^{1.1} \\
D_{f}^{C l-S C C}=134928.285
\end{array}
$$

Based on calculation Df above, the total damage factors may have value:

$$
\begin{aligned}
& D_{f-\text { total }}=D_{f}^{\text {thin }}+D_{f}^{C l-S C C} \\
& D_{f-\text { total }}=134928.385
\end{aligned}
$$

After determining gff total and Df total, the last factor that affects of PoF value is management system factor (FMS). As mention before, FMS is used to adjust gff for differences in process safety management systems [5]. The API 581 recommends the scale for converting management systems factor on the assumption of the “average" plant, would score $50 \%$ on the management systems evaluation. Therefore, the PoF may be determined:

$$
\begin{aligned}
P o F_{\text {tube }}^{g f f} & =g f f \cdot D_{f}(t) \cdot F_{M S} \\
P o F_{\text {tube }}^{g f f} & =2.065
\end{aligned}
$$

Figure 2 shows the linear value of $P o F_{\text {tube }}^{g f f}$ in a certain of service time. From the graph, it is shown that the $P o F_{\text {tube }}^{g f f}$ changes proportionally to the value of time. It

\begin{tabular}{|c|c|c|c|c|c|c|c|c|c|c|c|c|c|}
\hline \multirow{3}{*}{$S_{V I}$} & \multicolumn{13}{|c|}{ Inspection Effectiveness } \\
\hline & \multirow{2}{*}{$\mathbf{E}$} & \multicolumn{4}{|c|}{1 Inspection } & \multicolumn{4}{|c|}{2 Inspections } & \multicolumn{4}{|c|}{3 Inspections } \\
\hline & & D & C & B & A & D & $\mathrm{C}$ & B & A & D & $\mathrm{C}$ & B & $\mathbf{A}$ \\
\hline 1 & 1 & 1 & 1 & 1 & 1 & 1 & 1 & 1 & 1 & 1 & 1 & 1 & 1 \\
\hline 10 & 10 & 8 & 3 & 1 & 1 & 6 & 2 & 1 & 1 & 4 & 1 & 1 & 1 \\
\hline 50 & 50 & 40 & 17 & 5 & 3 & 30 & 10 & 2 & 1 & 20 & 5 & 1 & 1 \\
\hline 100 & 100 & 80 & 33 & 10 & 5 & 60 & 20 & 4 & 1 & 40 & 10 & 2 & 1 \\
\hline 500 & 500 & 400 & 170 & 50 & 25 & 300 & 100 & 20 & 5 & 200 & 50 & 8 & 1 \\
\hline 1000 & 1000 & 800 & 330 & 100 & 50 & 600 & 200 & 40 & 10 & 400 & 100 & 16 & 2 \\
\hline 5000 & 5000 & 4000 & 1670 & 500 & 250 & 3000 & 1000 & 250 & 50 & 2000 & 500 & 80 & 10 \\
\hline
\end{tabular}
means, the tube failure will increase steadily year by year. Table 6 shows the numerical values associated with PoF. From Table 6, the PoF category of $P o F_{\text {tube }}^{g f f}$ may be categorized as category " 5 ".

TABLE 5SCC DAMAgE FACTORS-ALL SCC MECHANISM 


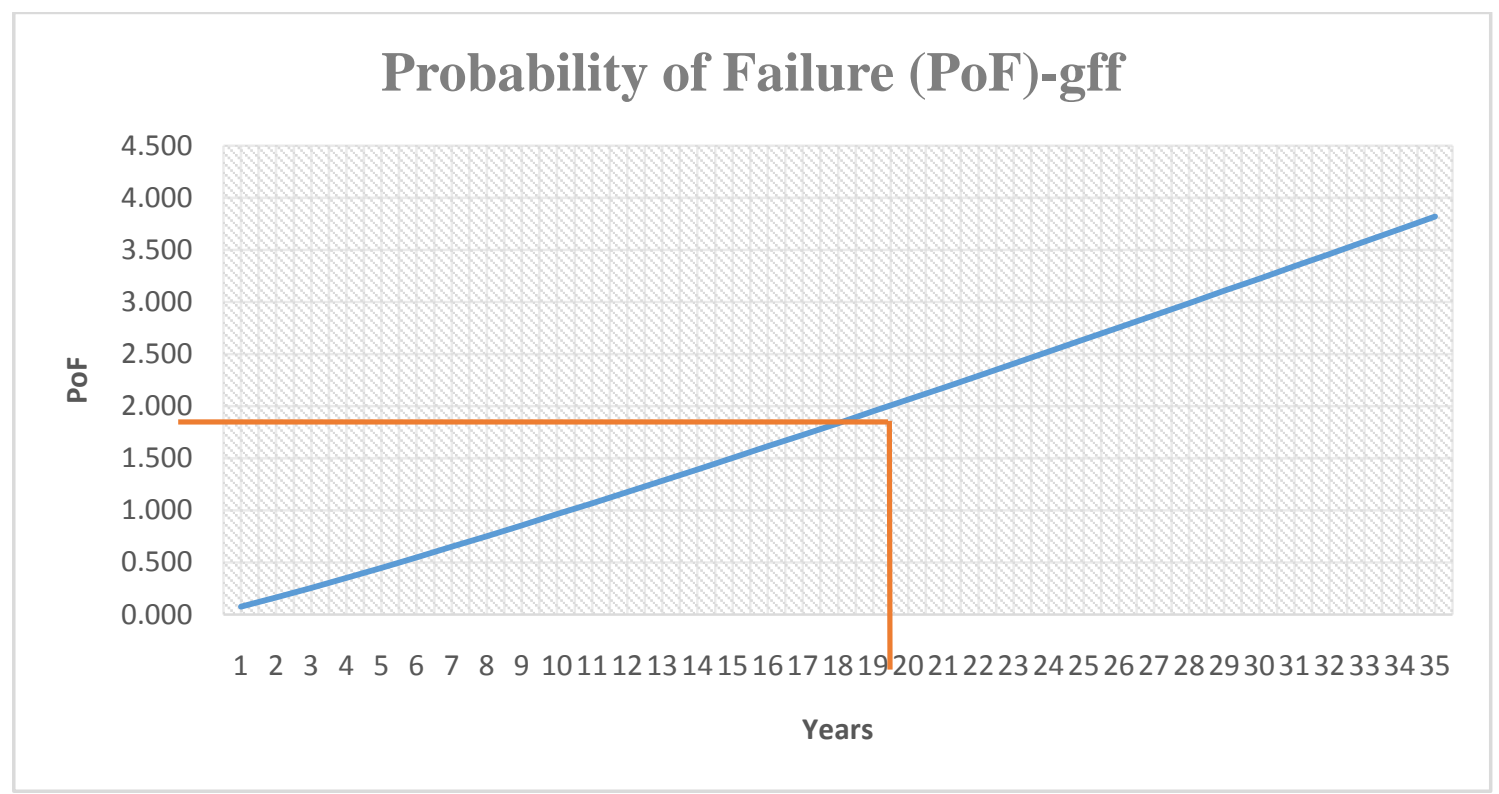

Figure 2. Probability of Failure (PoF) graph based on current study case

\begin{tabular}{|c|c|c|}
\hline \multicolumn{3}{|c|}{ Probability Category } \\
\hline Category & Probability Range & Damage Factor Range \\
\hline 1 & $\mathrm{PoF} \leq 3.06 \mathrm{E}-05$ & $\mathrm{D}_{\mathrm{f} \text {-total }} \leq 1$ \\
\hline 2 & $3.06 \mathrm{E}-05<\mathrm{PoF} \leq 3.06 \mathrm{E}-04$ & $1<\mathrm{D}_{\text {f-total }} \leq 10$ \\
\hline 3 & 3.06E-04 $<$ PoF $\leq 3.06 \mathrm{E}-03$ & $10<\mathrm{D}_{\mathrm{f} \text {-total }} \leq 100$ \\
\hline 4 & $3.06 \mathrm{E}-03<\mathrm{PoF} \leq 3.06 \mathrm{E}-02$ & $100<\mathrm{D}_{\text {f-total }} \leq 1000$ \\
\hline 5 & PoF $>3.06 E-02$ & $\mathrm{D}_{\text {f-total }}>1000$ \\
\hline
\end{tabular}

\section{Analysis of PoF Determination}

Based on case study above, there will be no difference of PoF determination result for heat exchanger tube. However, there are some interesting points which may be able to explore and give some new explanation about which one of those two PoF determination has the best value to use when conducting RBI assessment for heat exchanger tube.

$P o F_{\text {tube }}^{\text {wbl }}$ will give the exponential value of PoF based on its original formula. It showed that on year 14 to year 20, there were a significant PoF increase on heat exchanger tube. It was aligned with the practically considerations which assume an increasing number of static equipment with time and an increasing propensity to failure with age. Smith and Warwick [10] state on their research that there were $36 \%$ of failures are from components more than 10 years of age. However, from Figure 1, the PoF on early in design life period is contrary to some theoretical considerations, e.g. bathtub curve. Roesch said that the failure of infant region rate and at wear-out region are the highest. However, the most difficult to detect the failure between both of regions is in the infant region [11]. A quantitative models of infant mortality failures has been created by Wilkins to characterize failure distributions in all three phases of the bathtub curve. He said that the infant mortality is shown on year zero to year one has decreasing failure rate with total of 27 failures during this one year. However, there were only found 6 failures from one to ten years[12]. Therefore, when the heat exchanger are operated during normal region and wear-out region, the PoF determination for RBI assessment will be suitable when using $P o F_{t u b e}^{w b l}$ with some additional notes that the failure rate data should be available.

However, $P o F_{t u b e}^{w b l}$ did not determine the potential damage mechanism which influence the failures on the tube, susceptible material that will give higher probability of failure due to crack, number and effectiveness of inspection on heat exchanger tube. Therefore, it is also indicated that determination of $P o F_{t u b e}^{w b l}$ is suitable when the data of heat exchanger is insufficient. It will be useful to determine baseline inspection of heat exchanger tube when the heat exchanger is on normal region and still have no inspection data before.

In other side, $P o F_{\text {tube }}^{g f f}$ will give the linear value of PoF. The infant region of $P o F_{\text {tube }}^{g f f}$, year one to year four, shows that there were an accelerated number of PoF during that region. However, the highest value of increasing PoF is shown during year zero to year one. 
Moltoft said that the infant mortalities on the equipment will fail typical before 200 hours and often very early between 10 and 20 hours with percentage of 99.9\% [13]. $P o F_{\text {tube }}^{g f f}$ value will increase steadily during year five to year twenty five. It may contrary to general theory of bathtub curve, especially on wear-out region. Wear-out failure distributions usually appear on accelerated conditions which are intended to speed up time without generating new or anomalous failure mechanisms [11]. Therefore, the determination of $P o F_{\text {tube }}^{g f f}$ is not suitable when the heat exchanger operated between final phase of normal and wear-out region. The consideration is in the normal region, there were many inspection and maintenance data that will detect the possible damage mechanisms occurs in the heat exchanger. However based on the generic formula on API 581, determination of $P o F_{\text {tube }}^{g f f}$ did not consider the mitigation action of the tube when it is plugged as a temporary mitigation action.

Basically both PoF determination, $P o F_{\text {tube }}^{w b l}$ and $P o F_{\text {tube }}^{\text {gff }}$, have advantages and disadvantages when we will choose to determine which PoF that the most suitable to be used for RBI assessment for heat exchanger. However, this study give the suggestion for the next research to use the combination of both PoF determination with some percentage factor to achieve the optimum PoF value based on the actual condition. It requires a deeper analysis to consider the percentage value for each PoF determination.

\section{CONCLUSION}

API 581 has given two type of PoF determination. Both of PoF determination have their own advantages. $P o F_{\text {tube }}^{\text {wbl }}$ is more suitable to use when RBI analysis is conducted to determine the baseline inspection. Despite, $P o F_{\text {tube }}^{w b l}$ will be very useful when the owner-user gives the failure rate data without considering the potential damage mechanisms that occurs in the tube such as total of plugging tube, failure time, number of leakages tube, etc. However, $\mathrm{PoF}_{\text {tube }}^{g f f}$ determination requires more complex data. The owner-user should provide inspection history data, design and operating of heat exchanger data, contain of service fluid in the tube, and also the safety management issue on their plant. Therefore, $P o F_{\text {tube }}^{g f f}$ is suitably used when the heat exchanger already in-service and having comprehensive inspection and maintenance data.

\section{REFERENCES}

[1] J. Corte, J. Rebello, M. Areiza, S. Tavares and M. Araujo, "Failure analysis of AISI 321 tubes of heat exchanger," Engineering Failure Analysis, vol. 56, pp. 170-176, 2015.

[2] H. Peltola and M. Lindgren, "Failure analysis of a copper tube in a finned heat exchanger," Engineering Failure Analysis, vol. 51, pp. 83-97, 2015.

[3] API, "Risk Based Inspection-API Recommended Practice 580," 2016.
[4] D. Priyanta, "The Development of Equipment Criticality Analysis (ECA) Protocols of Offshore Carbon Steel Static Mechanical Equipment," Asian Journal of Applied Sciences, vol. 4, no. 06, pp. 1258-1266, 2016.

[5] API 581, Risk-based Inspection Methodology-third edition, American Petroleum Institute, 2016.

[6] R. Shilling, M. Rudy and T. Rudy, "Risk-Based Design Margin Selection for Heat Exchangers," in Proceedings of International Conference on Heat Exchanger Fouling and Cleaning VIII, Schladming, Austria, 2009.

[7] M. P. Schwartz, "Four Types of Heat Exchanger Failures," ITT Bell \& Gosset.

[8] R. A. Al-Badawi and M. N. Abdullah, "Tube Damage Mechanism and Analysis in Feed Water Heaters," Journal of Engineering and Development, vol. 16, pp. 261-272, 2012.

[9] API, API 571-Damage Mechanisms Affecting Fixed Equipment in the Refining Industry, Washington: American Petroleum Institute, 2011.

[10] T. Smith and R. Warwick, "A Survey of Defects in Pressure Vessels in the UK for the Period 1962-1978 and its Relevance to Nuclear Primary Circuits," International Journal Pressure Vessels and Piping, vol. 11, pp. 127166, 1983.

[11] W. J. Roesch, "Using a new bathtub curve to correlate quality and reliability," Microelectronics Reliability, vol. 52, pp. 2864-2869, 2012.

[12] D. J. Wilkins, "The Bathtub curve and Product Failure Behavior-Part One," [Online]. Available: http://www.weibull.com/hotwire/issue21/hottopics21.htm. [Accessed 2002 2018].

[13] J. Moltoft, "Behind the "Bathtub" Curve A New Model and Its Consequences," Microelectron Reliability, vol. 23, pp. 489-500, 1983. 